\title{
Foreword to the contributions of the 3rd International Acid-Base Symposium, Smolenice Castle, Slovakia, 2018
}

\author{
Jürgen Vormann ${ }^{1} \cdot$ Tanja Werner $^{2}$
}

(C) The Author(s), under exclusive licence to Springer Nature Limited 2020

In June 2018, scientists from 12 countries met in the beautiful location of the Smolenice Castle, Slovakia, for the 3rd International Acid-Base Symposium, Nutrition-Health -Disease. This supplement provides access to presentations held at that conference. In a stimulating atmosphere, the various aspects of acid-base metabolism were discussed and it was agreed that this-partly forgotten-aspect of human nutrition must be given more attention as balancing acids and alkalines in our body significantly contribute to general well-being.

Environmental pollution especially in northern Europe has long been connected to the problem of "acid rain." Whereas care for the environment could improve the ecological quality in that area, it has been neglected that-in analogy - too much acidity either through insufficient diet or metabolic causes could impair human health as well. Only in recent years this has been acknowledged, and pathophysiological mechanisms were further investigated to understand how too much acidity is connected to various diseases in our societies.

For many years considerations concerning importance of acid-base balance for human health in medicine and nutritional sciences were centered on effects induced by changed blood $\mathrm{pH}$. Blood acidosis or alkalosis normally does not occur or, if it happens, needs intensive medical treatment. It is only since few years that dietary induced so called "mild metabolic acidosis" or "latent acidosis" is recognized as being a result of western eating habits and might predispose for various diseases. In this condition blood $\mathrm{pH}$ is kept fairly constant; however, buffering capacity and $\mathrm{pH}$ in other

Jürgen Vormann

vormann@ipev.de

1 IPEV - Institute for Prevention and Nutrition, Adalperostr. 37, D-85737 Ismaning, Germany

2 NuOmix Research k.s., Applied Nutriomic Research, Bratislava, Slovakia compartments than blood might be changed. After development of reliable estimates for dietary acid load by Remer and Manz [1], these food tables for the potential renal acid load allowed to investigate the correlations between dietary intake of acids and bases with disease risks in large epidemiological studies.

Effects of changed acid-base balance and local acidosis with regard to the skeletal system are now known much more in detail and cellular mechanism concerning activity of osteoclasts and osteoblast have been elucidated. Furthermore, the connection of dietary acid load and sarcopenia in the elderly is recognized. Especially in our aging societies taking more care for a balance between acidic and alkaline foods will be a valuable tool to avoid loss of bone and muscle mass, which sometimes is-falsely-regarded as being unavoidable in aged persons.

Associations of dietary acid load with increased blood pressure or diabetes type 2 in observational studies have been published during recent years. Many of these interactions might have been overlooked in earlier investigations as reliable and easy to determine biomarkers were not available. By using $24 \mathrm{~h}$ urine samples and determining net acid excretion and net acid excretion capacity, more sensitive tools have been developed. With these methods already in children, a relation of body fatness and blood pressure with the acid burden of the kidneys could be observed.

Another important factor for kidney health is uric acid. Increased levels contribute not only to the risk of gout but also to the decline in renal function. Excretion of uric acid is reduced by dietary acid load and increased by alkalization.

It is known since long that metabolic acidosis is a risk factor for chronic renal failure. Increased dietary acid load will stimulate the need for renal ammonia excretion. Ammonia has some nephrotoxic potential and reduced need for ammonia production might avoid kidney function decline. Limiting or buffering dietary acid load in children with chronic renal failure improved metabolic acidosis. Dietary or pharmacological interventions to increase buffering capacity will improve kidney health. 
Alkalization might also beneficially influence the risk for type 2 diabetes. Even though in nondiabetics an effect of alkaline supplementation on glucose homeostasis could not be observed, it was shown in a placebo-controlled trial that alkaline supplementation improved oxidative carbohydrate metabolism in persons subjected to a dietary acid load.

Evolving evidence connects cancer progression to local acidosis. It is therefore tempting to speculate that increasing local or systemic buffering capacity could be a valuable tool in cancer treatment or prevention.

All these aspects of acid-base balance have been discussed in the Symposium and are covered in this publication giving the reader a thorough overview on the current knowledge on this important aspect of nutrition.

Funding This article is published as part of a supplement sponsored by NuOmix Research k.s. The conference was financially supported by
Protina Pharmazeutische GmbH, Germany, and SIRIUS GmbH, Germany, and organized by NuOmix Research k.s. Neither company had any role in writing of the manuscript.

\section{Compliance with ethical standards}

Conflict of interest JV has received consulting fees from Protina Pharmazeutische GmbH. JV and TW own equity in NuOmix Research k.s.

Publisher's note Springer Nature remains neutral with regard to jurisdictional claims in published maps and institutional affiliations.

\section{References}

1. Remer T, Manz F. Potential renal acid load of foods and its influence on urine pH. J Am Diet Assoc. 1995;95:791-7. 